\title{
Estudio Comparativo de los Servicios de Mantenimiento Utilizando Técnicas de Minería de Datos
}

\author{
A comparative study of maintenance services using the data-mining \\ technique
}

Antonio M. Cruz ${ }^{1}$, Wilmer A. Aguilera-Huertas² y Darío A. Días-Mora²

1 Escuela de Medicina, Universidad del Rosario. Bogotá D.C., Colombia. Antonio.cruz43.@urosario.edu.co 2 Universidad Manuela Beltrán. Bogotá. D. C, Colombia.

Recibido 7 Enero 2009/Enviado para Modificación 9 Julio 2009/Aceptado 14 Julio 2009

\section{RESUMEN}

Objetivos: Comparar la calidad del servicio de mantenimiento de dos entidades de salud. Una posee los servicios de mantenimientos tercerizados y la otra propios. El indicador de calidad bajo estudio es el tiempo de cambio de estado. Estudiar el comportamiento de la productividad del servicio de salud versus la disponibilidad. Materiales y Métodos. Se realizó un levantamiento del inventario de los equipos médicos y no médicos en dos servicios de diálisis ubicados en dos instituciones de salud. Ambas de tercer nivel. Estos poseen características similares en cuanto a las unidades de dializado y equipos médicos. Cada una de ellas posee 16 unidades para la atención a pacientes y 92 equipos médicos. La diferencia consiste en el servicio de mantenimiento; uno es tercerizado mientras que el otro es propio. Se recolectaron las órdenes de trabajo de mantenimiento por un período de 6 meses y se calcularon los indicadores: Tiempo de Respuesta, Duración Real, Tiempos Perdidos y Tiempo de cambio de estado (TAT) para cada orden de trabajo. Se construyó un predictor para la variable TAT (variable dependiente) en función de las otras variables.

Resultados. La calidad del servicio de mantenimiento por la entidad que posee su propio personal es mejor que la entidad con servicios tercerizados. El indicador TAT es como promedio de 2,95 horas para la entidad con mantenimiento propio, mientras que para la otra entidad es de 3,4 horas.

Conclusiones: El comportamiento de la productividad del servicio versus la disponibilidad resultó ser de tipo positiva lineal.

Palabras Clave: Servicio de mantenimiento e ingeniería en hospital, modelos lineales, Gerencia. 


\section{ABSTRACT}

Objectives The main goal in this research was comparing two hospitals' maintenance service quality. One of them had a contract service; the other one had an in-house maintenance service.

Materials and methods The authors followed the next stages when conducting this research: domain understanding, data characterisation and sample reduction, insight characterisation and building the TAT predictor. Multiple linear regression and clustering techniques were used for improving the efficiency of corrective maintenance tasks in a clinical engineering department (CED). The indicator being studied was turnaround time (TAT).

Results The institution having an in-house maintenance service had better quality indicators than the contract maintenance service.

Conclusions There was lineal dependence between availability and service productivity.

Key Words: Maintenance and engineering, hospital, regression analysis, biomedical technology, decision support system, management (source: MeSH. NLM).

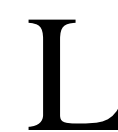

a ingeniería clínica es una de las ramas de la ingeniería biomédica, según el Colegio Americano de Ingeniería Clínica ( por sus siglas en inglés ACCE), el ingeniero clínico es un especialista que “....apoya y contribuye a mejorar la calidad del servicio de salud que se brinda a los pacientes a través de la aplicación de competencias adquiridas, relacionadas con el campo de la ingeniería y la gestión de la tecnología..." (1).

Uno de los procesos asociados a la gestión de tecnología es la gestión de los contratos de servicios de mantenimiento u "outsourcing. El outsourcing es una estrategia empresarial por medio de la cual una organización delega formalmente funciones misionales y no misionales a un tercero (2).

Algunos trabajos han reportado que la contratación de los servicios de mantenimiento en las instituciones de salud oscila entre un 15 y un $20 \%$ del total del inventario de equipos (3-5). También se ha reportado que se pueden reducir los gastos que implican los contratos de servicios de mantenimiento hasta en un $20 \%$ gracias a un adecuado control y seguimiento del desempeño de los proveedores de estos servicios. (6). Las variables más usadas para medir y evaluar la calidad del servicio de mantenimiento, ya sea tercerizado o no son: la disponibilidad, número de reparaciones adicionales por año, duración de cada intervención (DR), tiempos de respuesta (RT), reparaciones realizadas correctamente la primera vez y reparaciones hechas en el tiempo recomendado, tiempos de cambio de estado (TAT) y tiempos perdidos (TP) (7). 
El problema de la evaluación y control de las actividades de mantenimiento mediante el cálculo de los indicadores técnico-económicos por métodos sistemáticos y universales se observa que aun no se ha abordado con suficiente profundidad en la literatura. En el caso específico de Colombia, se ha reportado que "... En Colombia no se han realizado profundos estudios sobre este tema, las únicas indagaciones que se han hecho se refieren en alguna medida a la importancia que esta estrategia tiene entre las Instituciones Prestadoras de Servicios de Salud en Colombia....”(2)

Un análisis bibliográfico realizado constató esta falencia. Los trabajos encontrados tratan de aplicar la Teoría de Atributos Múltiples (por sus siglas en ingles MAUT) para resolver el problema anteriormente planteado (8-11).

Otros autores han aplicado métodos más universales para intentar resolver el problema de la evaluación del desempeño de los proveedores de servicio. Se ha encontrado que se han usado métodos de inteligencia artificial para este propósito (13), específicamente Lógica Difusa (14).

Este trabajo intenta llenar el vacío que existe en los métodos de evaluación de la calidad de los proveedores de serviciode mantenimiento (outsourcing o tercerización de los servicios de mantenimiento). Este se realizará mediante la creación de un nuevo indicador numérico usando métodos y herramientas sistemáticas (las técnicas de regresión lineal múltiple).

El método de construcción del indicador garantiza que el modelo sea transferible y aplicable a todas las instituciones de salud en las cuales se desee implementar dicho indicador.

La investigación tiene como objetivo principal:

- Obtener un nuevo indicador numérico que permita medir o caracterizar el desempeño de los proveedores de servicio de mantenimiento a las instituciones de salud que tienen servicios tercerizados y las que no lo tienen.

- Realizar un estudio del comportamiento de la relación entre el indicador productividad del servicio de salud y la disponibilidad.

Las preguntas de investigación son:

¿Cómo se relacionan las variables: tiempos perdidos(TP), tiempo de respuesta (TR), la duración de las intervenciones de mantenimiento (DR) con el tiempo de cambio de estado del equipamiento (TAT) para equipos que son atendidos por medio de contratos de servicios de mantenimiento y los que no. 
¿Cuál es el comportamiento de la productividad del servicio de salud en función del indicador TAT?

\section{MATERIALES Y METODOS}

La metodología llevada a cabo para la realización fue la siguiente.

El estudio se realizó en dos unidades renales pertenecientes a la Fundación Renal, con sede en la ciudad de Bogotá. Esta entidad ofrece servicios de diálisis peritoneal y nefrología, entre otros, en varios hospitales del país, con un volumen de secciones de diálisis del orden de las 6 556. Esta entidad arrendó los espacios ubicados en cada institución de salud para ofrecer los servicios de diálisis. Una unidad renal está ubicada en el Hospital Confamiliar (50 camas) en la ciudad de Cartagena; y la otra en la Clínica el Parque (60 camas) en la Ciudad de Armenia. Ambas son instituciones de tercer nivel. Cada servicio renal posee 16 unidades o módulos para la atención a pacientes y 92 equipos en el inventario, cuyo valor es de \$ 1221857358 (US \$ 581836 aproximadamente) y \$ 1148336255 (US \$ 546826 aproximadamente) para el caso de Armenia y Cartagena respectivamente. La única diferencia entre ambas es el tipo de servicio de mantenimiento; en una el servicio es tercerizado mientras que en la otra es propio, en adelante denotadas como "P" (unidad renal en Cartagena) y " $\mathrm{T}$ " (unidad renal en Armenia) respectivamente.

Para demostrar las similitudes entre las instituciones de salud en términos de equipamiento se realizó un levantamiento del inventario de los equipos médicos y no médicos. Mostrándose los resultados en tablas comparativas (Tabla 1).

Se recolectaron las órdenes de trabajo producto del mantenimiento realizado durante un periodo de 6 meses y se calcularon los indicadores de calidad del servicio de mantenimiento: TR, DR, TP y TAT para cada orden de trabajo.

Se construyó un predictor para la variable TAT (variable dependiente) en función de las variables TR, DR, y TP (variables independientes), para cada tipo de servicio de mantenimiento.

Se estudió el comportamiento de la productividad del servicio de salud en función del indicador TAT calculado con el predictor para cada tipo de servicio de mantenimiento. 


\section{RESULTADOS}

La Tabla 1 muestra los resultados obtenidos producto del levantamiento del inventario. De estas se pueden encontrar los siguientes patrones:

El equipamiento instalado en la institución con servicio de mantenimiento tercerizado es más "longevo" que el que tiene servicio propio. Siendo como promedio la razón TE/VU en el primero de 1,034; mientras que en el segundo es de 0,79 (ver Columna 3 y 7 Tabla 1 )

Tabla 1. Caracterización general del inventario del inventario en las instituciones bajo estudio

\begin{tabular}{|c|c|c|c|c|c|c|c|c|}
\hline \multirow[b]{2}{*}{$\begin{array}{c}\text { Tipos de } \\
\text { Equipos }\end{array}$} & \multicolumn{4}{|c|}{ Servicio tipo "T" } & \multicolumn{4}{|c|}{ Servicio tipo "P" } \\
\hline & $\begin{array}{c}\# \\
\text { Equipo }\end{array}$ & TENU & $\begin{array}{c}\text { Valor } \\
\text { inventario }\end{array}$ & $\begin{array}{c}\% \text { del } \\
\text { valor } \\
\text { Inventario }\end{array}$ & $\begin{array}{c}\# \\
\text { Equipo }\end{array}$ & TE/VU & $\begin{array}{c}\text { Valor } \\
\text { inventario }\end{array}$ & $\begin{array}{c}\% \text { del } \\
\text { valor } \\
\text { Inventario }\end{array}$ \\
\hline Terapia & 18 & 0,8 & 664500000 & 54,4 & 17 & 0,7 & 586200000 & 51 \\
\hline Monitoreo & 7 & 0,83 & 89008484 & 7,3 & 5 & 1,2 & 22015958 & 2 \\
\hline Diagnástico & 15 & 1,44 & 33900000 & 2,8 & 13 & 0,7 & 15260000 & 1,3 \\
\hline $\begin{array}{l}\text { Otros } \\
\text { médicos }\end{array}$ & 29 & 1.04 & 184446000 & 15,1 & 25 & 0,8 & 227351738 & 19,8 \\
\hline $\begin{array}{l}\text { Equipos de } \\
\text { Osmosis } \\
\text { Inversa }\end{array}$ & 16 & 1,07 & 89992761 & 7,4 & 14 & 0,4 & 92405672 & 8 \\
\hline No médicos & 7 & 1,03 & 160010113 & 13 & 18 & 0,8 & 205102887 & 17,9 \\
\hline Total & 92 & & 1221857358 & 100,0 & 92 & & 1148336255 & 100,0 \\
\hline
\end{tabular}

* Terapia: Maquinas de hemodiálisis, desfibriladores, succionadores; Equipos de Osmosis e instalación de apoyo a las maquinas de diálisis: Filtros de varios tipos y sistema de osmosis; Monitoreo: Monitores de ECG, Tensiómetros; Diagnóstico: Ultrasonido; Otros médicos: Carros de medicamentos, carros de paro y sillas para dializado, negatoscopio, lámparas de UV; No médico: Aires acondicionados, elevadores

Los equipos de terapia, o sea los involucrados en el tratamiento de diálisis y los de apoyo a estas máquinas (Equipos de Osmosis Inversa), para ambas instituciones consumen el $61,8 \%$ en una caso y el $59,1 \%$ en el otro caso de valor total de inventario (columnas 5 y 9 de la Tabla 1). Observe la similitud de los valores, siendo la diferencia entre uno y otro caso de solo un $2,7 \%$

Un segundo resultado importante que arroja este estudio fue la construcción de un predictor utilizando un método de correlación lineal múltiple para el indicador TAT para cada tipo de servicio de mantenimiento en las instituciones bajo estudio. La herramienta empleada para la construcción de este fue $\mathrm{WEKA}^{\circledR}$, en su versión 3.4 .7 (14). El predictor fue construido empleando el algoritmo SMOREG (15). El predictor tiene la forma que se expresada en la Ecuación 1. En la Tabla 2 se muestran los valores de los coeficientes de cada una de las variables que están presentes en los predictores para cada tipo de servicio en cuestión. 
$T A T=b_{o}+b_{1} * T R+b_{2} * D R+b_{3} * T P$

Donde:

$\mathrm{b}_{\mathrm{o}}, \mathrm{b}_{1}$. $\mathrm{b}_{2}$ y $\mathrm{b}_{3}$ : son coeficientes de regresión

TR: es el tiempo de respuesta en horas (4-5)

DR: duración real de la intervención de mantenimiento en horas (4-5)

TP: tiempos perdidos en horas (4-5)

TAT: tiempo de cambio de estado en horas (4-5)

Tabla 2. Construcción de los predictores TAT para cada uno de los tipos de servicios en las instituciones de salud

\begin{tabular}{|c|c|c|c|c|c|}
\hline \multicolumn{3}{|c|}{ Servicio tipo "T" } & \multicolumn{3}{|c|}{ Servicio tipo "P" } \\
\hline Coeficiente & Variables & Valor & Coeficiente & Variables & Valor \\
\hline$b_{o}$ & & -0.006 & $b_{0}$ & & -0.0247 \\
\hline$b_{1}$ & TR & +0.1287 & $b_{1}$ & TR & +0.3155 \\
\hline$b_{2}$ & DR & +0.156 & $b_{2}$ & DR & +0.3393 \\
\hline$b_{3}$ & TP & +0.9282 & $b_{3}$ & TP & +0.5526 \\
\hline \multicolumn{3}{|c|}{ Validación Cruzada } & \multicolumn{3}{|c|}{ Validación Cruzada } \\
\hline \multicolumn{2}{|c|}{ Coeficiente de Correlación } & 0,98 & \multicolumn{2}{|c|}{ Coeficiente de Correlación } & 0,96 \\
\hline \multicolumn{2}{|c|}{ Error Absoluto Medio } & 0,0228 & \multicolumn{2}{|c|}{ Error Absoluto Medio } & 0,0094 \\
\hline \multicolumn{2}{|c|}{$\begin{array}{c}\text { Raíz cuadrada de la media } \\
\text { del error }\end{array}$} & 0,0692 & \multicolumn{2}{|c|}{ Raíz cuadrada de la media } & 0,022 \\
\hline \multicolumn{2}{|c|}{ Error Absoluto (\%) } & 0,6 & \multicolumn{2}{|c|}{ Error Absoluto (\%) } & 0,4 \\
\hline \multicolumn{2}{|c|}{$\begin{array}{l}\text { Raíz cuadrada del Error } \\
\text { Absoluto }(\%)\end{array}$} & 0,9 & \multicolumn{2}{|c|}{$\begin{array}{c}\text { Raíz cuadrada del Error } \\
\text { Absoluto (\%) }\end{array}$} & 0,62 \\
\hline
\end{tabular}

Con los coeficientes del predictor y los valores de las variables independientes, se calcula el TAT y la disponibilidad para el período analizado. El número de horas de operación teóricas de cada equipo se calcula estimando la cantidad de tratamientos a realizar multiplicado por la duración promedio de cada tratamiento o procedimiento médico.

La Figura 1 parte a) y b) muestra el comportamiento de la disponibilidad del equipamiento en función de la productividad del servicio médico. Denotándose una tendencia correlacional positiva para ambos casos. El cálculo arrojó un coeficiente de correlación de 0,81 , un $\mathrm{R}^{2}$ ajustado de 0,7 y un error típico de 0,004 para la institución que posee servicios de mantenimiento contratados con entidad externa. Para el caso de la otra institución, el coeficiente de correlación fue de 0,8 , un $\mathrm{R}^{2}$ ajustado de 0,5 y un error típico de 0,002 .

La calidad del servicio de mantenimiento ofrecido por la entidad que posee su propio personal contratado fue de mejor calidad que la entidad que posee sus servicios tercerizados. El indicador TAT es como promedio de 2,9 horas 
para la entidad con servicios de mantenimiento propios, mientras que para la otra entidad este valor de es 3,4 horas, o sea 1,2 veces mejor. Lo mismo ocurre para el caso del indicador TP y DR de las intervenciones. Siendo el TP y DR 2,9 y 1,1 veces mejor para el caso de la institución que posee servicios de mantenimiento propios.

Figura 1. Análisis de regresión de la variable disponibilidad versus productividad

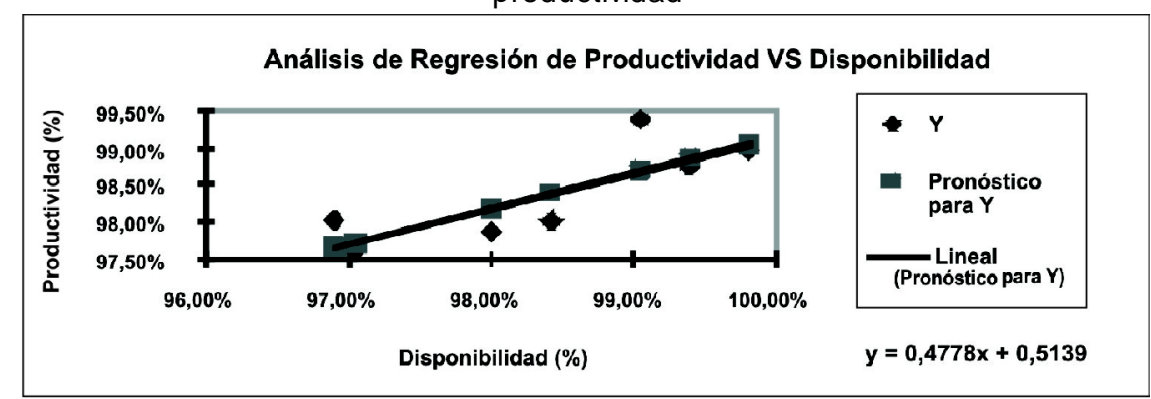

a. Institución con servicios de mantenimiento contratados

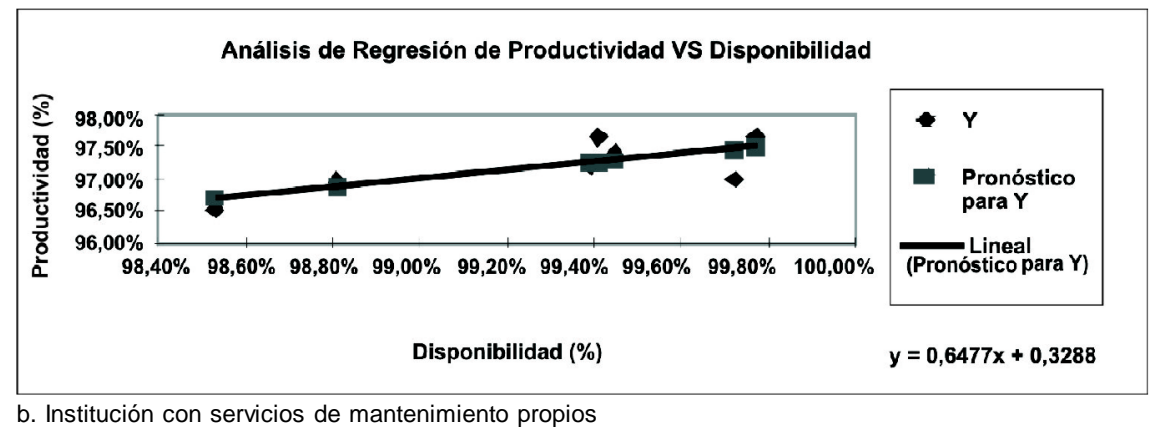

b. Institución con servicios de mantenimiento propios

\section{DISCUSIÓN}

La determinación del predictor en si mismo permite determinar dos cosas; por una lado calcular los valores de TAT y llegar a predecir o anticipar el comportamiento futuro de este indicador para cada tipo de servicio; y por el otro permite determinar a través de los coeficientes $\left(b_{1}, b_{2} y_{3}\right)$ cuales son las variables que más dependen fuertemente del valor final del indicador TAT para cada uno de los casos estudiados. De esta manera se puede ver que: 
- Para la institución que tiene los servicios de mantenimiento contratados o tercerizados, el factor o variable que más influye en el valor del indicador TAT es la variable TP, $\mathrm{o}$ sea, el tiempo perdido. Esto implica que el proveedor de servicios a pesar de tener tiempos de respuestas y de reparación aceptables (coeficientes de $+0,1$ para las variables DR y TR), pierde mucho tiempo en la logística de la ejecución de los trabajos, ya sea por una mala gestión de piezas de repuestos o por otras causas indeterminadas. Este resultado es preocupante por cuanto se pierde el $46 \%$ del tiempo en actividades improductivas.

- En cambio, para el caso de la institución que tiene los servicios de mantenimiento propios, existe un mayor equilibrio en las variables que influyen en el indicador de TAT. Se tiene un coeficiente de +0.3 para DR y TR y $+0,6$ para TP. Estos coeficientes permiten llegar a la conclusión de que el valor de TAT tiene una dependencia más fuerte en las variables DR y TR, o sea los tiempos para reparar y para llegar al lugar de la reparación, pero se pierde mucho menos tiempo en terminar los trabajos en comparación con la otra institución.

La correlación entre de la productividad versus la disponibilidad obtenida resultó ser mejor para el caso de la entidad que posee los servicios de mantenimiento contratados $(0,8$ versus 0,7$)$. Sin embargo, la dependencia entre la disponibilidad del equipamiento y la productividad del servicio resultó ser más fuerte para el caso de la entidad que posee los servicios propios.

Hay factores de índole asistencial afectan a la productividad del servicio. Estos son los casos de ausencia del personal asistencial, falta de insumos, interrupción eléctricas, de agua, etc. Este resultado ha sido obtenido de esta manera gracias a que estas causas de interrupción del servicio han sido descartadas en el estudio que se ha efectuado.

La investigación presenta limitaciones como por ejemplo, realizar nuevos cálculos del predictor con otros algoritmos implementados en la herramienta WEKA con el objetivo de determinar mejores niveles de exactitud. Otra limitación importante fue la no disponibilidad de información de los equipos, debido a la ausencia de registros históricos de datos de los mismos.

De las conclusiones de este trabajo se puede plantear que:

- Se evidencia que la calidad del servicio de mantenimiento ofrecido por la entidad que posee su propio personal contratado es de mejor calidad que la entidad que posee sus servicios tercerizados. 
- El indicador TAT es como promedio de 2,9 horas para la entidad con servicios de mantenimiento propios, mientras que para la otra entidad este valor de es 3,4 horas, o sea 1,2 veces mejor.

- El comportamiento de la productividad del servicio versus la disponibilidad resultó ser de tipo positiva lineal. $\mathrm{O}$ sea, a mejor disponibilidad mejor productividad del servicio

Agradecimientos. A COLCIENCIAS por financiar el proyecto en la Convocatoria: 459/08, Proyectos investigación prioritaria en salud. A la Fundación Renal con sus unidades renales de las ciudades de Armenia y Cartagena por su apoyo y colaboración en la obtención de los datos necesarios para la realización de esta investigación.

\section{REFERENCIAS}

1. American College of Clinical Engineering [Internet]. Disponible en: http://www.accenet.org/ default.asp?page=publications\&section=presentations. Consultado Diciembre del 2008.

2. Ministerio de la Protección Social. Modelo de Evaluación y Gestión del Equipamiento Biomédico [Internet]. Disponible en: http://www.minproteccionsocial.gov.co/VBeContent/Library/documents/DocNewsNo14710DocumentNo5095.pdf. Consultado diciembre de 2008.

3. Cohen T. Benchmark indicators for medical equipment repair and maintenance. Biomedical Instrumentation and Technology, 1995; 29(4):308-320.

4. Cohen T. Benchmark indicators for medical equipment repair and maintenance: Parts I. Biomedical Instrumentation and Technology. 1995; 29(5):136-144.

5. Cohen T. Validating medical equipment repairs and maintenance metrics: Parts II. Biomedical Instrumentation and Technology. 1998; 32(2):136-144.

6. ECRI. In House servicing of X-ray and CT Equipment, Health Technology Management. 1993: Volume Winter: 34-45.

7. ECRI. Health Technology. Types of services their advantages and disadvantages, Special report on managing senvice contract, 1989; 3(4):39-21.

8. Almeida AT. Repair Contract Decision Model Through Additive Utility Function. Journal of Quality in maintenance in engineering, 2001;7(1): 42-48.

9. Page FH. Dominant strategy mechanisms for contract auctions with risk aversion and moral hazard. Journal: Game Theory, 1985; 23(1): 25-42.

10. Teixeira A. Repair contract decision model through additive utility function. Journal of Quality in Maintenance Engineering, 2001; 7(2):42-48.

11. Anderson TJ. Evaluating medical equipment senvice option. Instrumentation and Technology. 1989; 33(3):195-198.

12. James JP. Equipment Management Risk Rating System Based on Engineering Endpoints. Biomedical Instrumentation and Technology, 1999; 7(2): 115-120.

13. Cruz AM, Denis ER. A fuzzy inference system to evaluate contract service provider performance. Biomed Instrum Technol. 2005; 39(4):320-5.

14. Waikato University [Internet]. Disponible en: http://www.cs.waikato.ac.nz/ml/weka. Consultado Diciembre de 2008.

15. Alex J. Smola, Bernhard S. A Tutorial on Support Vector Regression. NeuroCOLT2 Technical Report Series - NC2-TR-1998-030, S.K. [Internet]. Disponivle en http://eprints.pascal-network.org/ archive/00002057/01/SmoSch03b.pdf. Consultado Diciembre de 2008.

16. Mosquera CG Disponibilidad y confiabilidad de sistemas industriales. $3^{\text {ra }}$ Edición, Valencia: Venezuela; 1995. 